\title{
National Identification and Authentication System (NIAS)
}

\author{
Dario Belić \\ Director of IT Development Service \\ ICT Division \\ Financial Agency - FINA \\ Ulica grada Vukovara 70, Zagreb, Croatia \\ dario.belic@fina.hr
}

\section{Summary}

NIAS is an information-technological system for the central identification and authentication of e-services users at the national level with the ability to use multiple types of credentials of various issuers which are assigned different levels of security authentication.

All owners of credentials are allowed by NIAS safe use of NIAS connected electronic services.

At the same time, providers of electronic services are released from the issuing of credentials and user identity management.

During the authentication process all subjects or their information systems communicate with the NIAS system exchanging XML messages according to the SAML 2.0 standard.

When transferring SAML messages between subjects and NIAS, confidentiality and integrity are secured using the mechanisms of the electronic signature of SAML messages and SSL encryption.

NIAS provides Single Sign-On and Single-LogOut functionalities. NIAS is designed on the principles of the EU STORK project (Secure idenTity acrOss boRders linKed) respecting the already existing practices and accepted standards to ensure that in the moment of electronic connectivity with EU members the process is performed as simply as possible.

Cross-border connecting of NIAS system is currently in progress, based on the STORK standards, and also the expansion to e-business.

Keywords: NIAS, Credentials, STORK, SAML, Authentication, Identification, service Provider, identity provider, OIB 